\title{
Lupus erythematosus panniculitis resistant to standard treatment, complicated with macrophage activation syndrome
}

\author{
Katarzyna Juczynska, Anna Wozniacka, Elzbieta Waszczykowska, Agnieszka Zebrowska
}

Department of Dermatology and Venereology, Medical University of Lodz, Lodz, Poland

Adv Dermatol Allergol 2017; XXXIV (3): 281-283

DOI: https://doi.org/10.5114/ada.2017.67852

Lupus erythematosus panniculitis (LEP) is an uncommon form of chronic cutaneous lupus erythematosus [1-3]. Terms LEP and lupus profundus were used interchangeably, however it is suggested that the term "lupus profundus" should be used when the subcutaneous inflammatory process is accompanied by overlying lesions typical for discoid lupus erythematosus (DLE) [4]. The LEP occurs more frequently in adult women. Typical lesions for the disease are deep-seated, indurated, firm nodules and plaques in locations rich in adipose tissue often healing with ulcerations, atrophic scars and/ or calcification $[5,6]$. There are no univocal criteria for LEP established. Histopathologic findings are reported to be crucial, however there is no agreement considering specific histopathological results [7, 8]. A positive lupus band test revealed in direct immunofluorescence studies, positive ANA test, leucopenia and hypocomplementemia stated in laboratory findings might be helpful. Differential diagnosis for LEP includes many diseases such as erythema nodosum, pancreatic panniculitis, morphea profunda, cold-panniculitis and, first of all, subcutaneous panniculitis-like T-cell lymphoma (SPTCL), which may be clinically identical with LEP $[9,10]$. Immunohistochemical analysis of cells constituting infiltrate is in most cases crucial to distinguish these two diseases. All known therapy strategies for LEP are based on clinical experience and single reported cases, they include antimalarials, immunomodulators, immunosuppressants, intravenous immunoglobulins (IVIG) and rituximab [4, 5, 8, 11, 12].

Macrophage activation syndrome (MAS) is a severe, life-threating hematologic condition and is classified in the group of secondary hemophagocytic lymphohistiocytosis (HLH). The MAS is caused by uncontrolled activation and proliferation of $\mathrm{T}$ lymphocytes and macrophages and is characterized by hemophagocytosis in bone marrow, hepatosplenomegaly, cytopenia, hyperferritinemia, hypofibrinogenemia, elevated liver cell enzymes, lymphadenopathy and fever [13]. Therapeutic options for the disease include high doses of steroids, cyclosporine A, intravenous immunoglobulins, etoposide, infliximab, rituximab and anakinra [14].

We present a case of a 40-year-old woman, who was admitted to the Department of Dermatology with deepseated, firm in consistency, subcutaneous nodules and plaques on the face, neck, neckline, arms and buttock with high grade fever and fatigue, with no lesions typical for DLE. Family and personal history of any autoimmune disease was negative. Laboratory results revealed anemia, leukopenia, and increased $\mathrm{C}$-reactive protein (CRP). There was no evidence of preceding trauma and an active bacterial or viral infection, including negative test for Ebstein-Barr virus (EBV). The titers of ANA were $1: 320$ and $1: 160$, respectively, anticardiolipin antibodies were negative. Serum examinations for liver, renal and thyroid function, glucose level, C3, C4, rheumatoid factor and urine analysis were all normal. Direct immunofluorescence examination revealed a negative lupus band test. Spleen and liver enlargement were detected in the computed tomography (CT) scan.

Three skin biopsies were performed and subjected to histopathological examination. The specimens revealed epidermis without any pathological changes, and in dermis perivascular and interstitial infiltrations of lymphocytes, histiocytes and miofibroblasts. In subcutaneous tissue, focal minor granulomas were present as well as a lobular pattern of inflammation consisting of lymphocytes, histiocytes and granulocytes. In order to differentiate between LEP and the lymphoproliferative process, there were immunohistochemical analyses performed, which revealed predominance of CD3+ CD4+ CD8+ CD56- granzyme B-, TIA 1- lymphocytes T and CD3+ CD8+ CD4- lymphocytes $T$ in infiltrates. On the basis of

Address for correspondence: Katarzyna Juczynska MD, Department of Dermatology and Venereology, Medical University of Lodz, 1 Hallera Sq. build. 6, 90-647 Lodz, Poland, phone: +48 603690 219, e-mail: juczynska@yahoo.com Received: 4.07.2016, accepted: 4.10.2016. 
clinical features, histopathologic, immunohistochemical and laboratory findings, the diagnosis of LEP was made and a lymphoma process was excluded. The patient was initially treated with oral steroid (prednisone), antimalarial (chloroquine) and cyclosporine A. There was no significant clinical improvement noted, existing indurations with edema enlarged and new nodules developed (Figure 1). Further therapy was based on chloroquine, prednisone and azathioprine. In the course of treatment, depressed atrophic scars, bruises and ulcerations covered with crusts appeared among the existing lesions and further indurations on the face and both arms were found. After failure of conventional therapeutic agents, the patient was treated with intravenous immunoglobulins in combination with oral prednisone (40-30 mg/ day) and chloroquine ( $250 \mathrm{mg} /$ day). There was a clinical improvement noted, no further nodules developed and the existing ones started to resolve with significant lipoatrophy (Figure 2). However, after the first following pulse, the patient revealed hyperferritinemia, hypofibrinogenemia in laboratory findings and significant progression of cytopenia and splenomegaly. Hemophagocytosis was present in the bone marrow, and hence the patient fulfilled diagnostic criteria for MAS. She has been treated for the disease in the Hematology Department.

The objective of this study is to report a rare case of a patient diagnosed with LEP with failure to respond to standard treatment, including oral steroid, antimalarial and immunosuppressive agents (cyclosporine A, azathio-

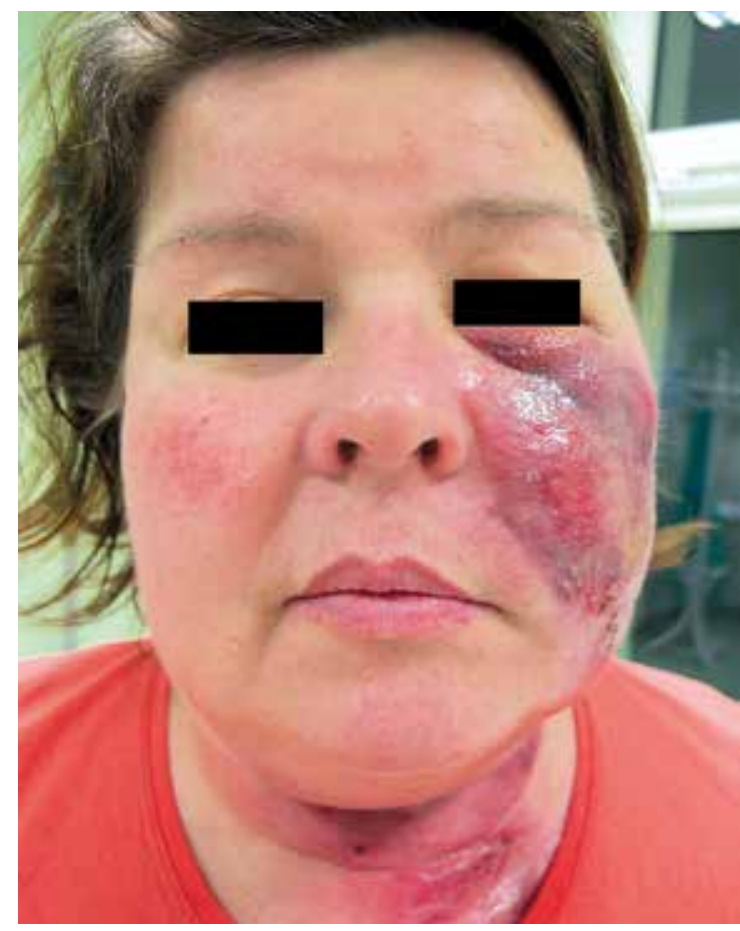

Figure 1. Inflammatory indurations with edema on the left cheek and neck, a new nodule on the right cheek prine), who responded to intravenous immunoglobulins, however developed MAS. Therapy strategies for LEP involving IVIG are based on single case reports [4, 11, 12]. The patient received immunoglobulins (Sandoglobulin P) in the initial pulse of $2 \mathrm{~g} / \mathrm{kg}$ of body weight, in combination with oral steroids and antimalarials, followed by $400 \mathrm{mg} / \mathrm{kg}$ body weight IVIG pulses at monthly intervals for a maximum of 6 months, in accordance with the regime reported by Goodfield et al. [11]. The strategy was successful, as after a starting pulse, a clinical improvement was noted sustained by the following pulse.

The MAS is a rare, however recently more often diagnosed complication of inflammatory and autoimmune diseases, viral infections (particularly EBV), lymphomas and immunosuppressive therapy among others [13, 14]. It is reported that MAS may associate with both benign and malignant types of panniculitis, e.g. cytophagic histiocytic panniculitis, LEP and SPTCL $[15,16]$. It may also develop as the onset or in the course of SLE, the incidence of such coexistence is about $0.9-4.6 \%[17,18]$. There are various hypotheses concerning the exact pathogenesis of MAS and its possible concomitance with autoimmune disorders. As suggested by Deji et al., common antibodymediated mechanisms might be responsible for both lymphocytic lobular panniculitis (as present in LEP) and hemophagocytic syndrome [16]. The other mechanisms taken into consideration in pathogenesis of reactive hemophagocytis in SLE are immune complex-mediated mechanisms and involvement of pro-inflammatory cyto-

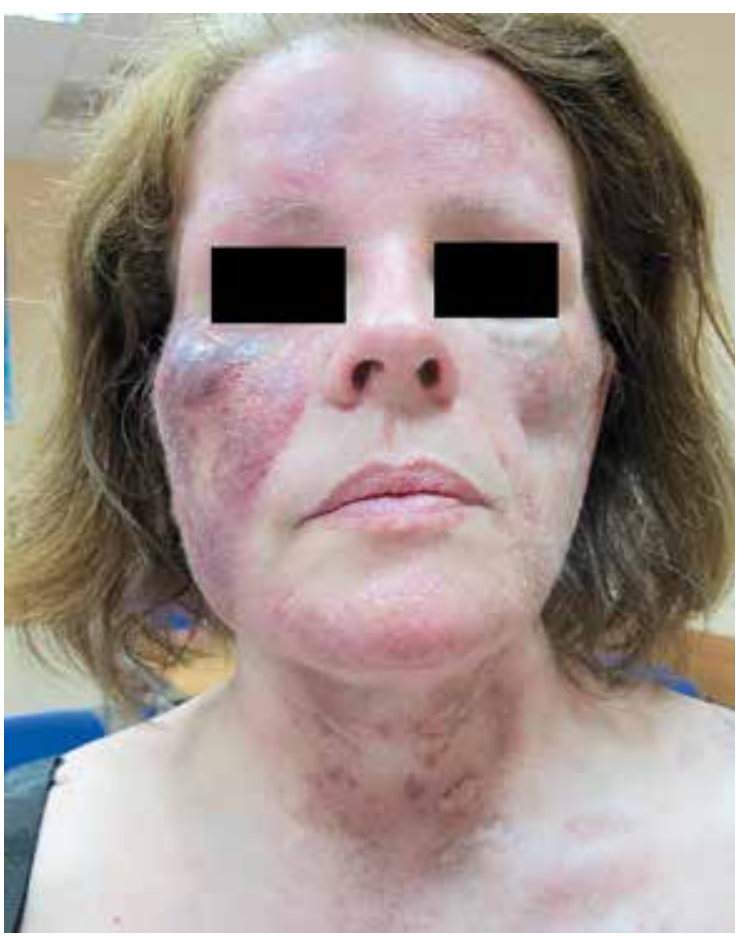

Figure 2. Healing phase with significant lipoatrophy 
kines, however all those hypotheses require further studies [18]. In the presented case, no evidence of infection (including EBV) and occurrence of MAS in the course of LEP, after introducing a new therapy (IVIG) indicate that MAS may be considered as a complication of the autoimmune condition, however it could be triggered by the immunosuppressive therapy as well.

\section{Conflict of interest}

The authors declare no conflict of interest.

\section{References}

1. Tuffanelli DL. Management of cutaneous lupus erythematosus. Clin Dermatol 1985; 3: 123-30.

2. Bednarek A, Bartoszak L, Samborski W. Case report on a patient with lupus panniculitis. Postep Derm Alergol 2015; 32: 59-62.

3. Szczęch J, Rutka M, Samotij D, et al. Clinical characteristics of cutaneous lupus erythematosus. Adv Dermatol Allergol 2016; 33: 13-7.

4. Espirito Santo J, Gomes MF, Gomes MJ, et al. Intravenous immunoglobulin in lupus panniculitis. Clin Rev Allergy Immunol 2010; 38: 307-18.

5. Martens PB, Moder KG, Ahmed I. Lupus panniculitis: clinical perspective from a case series. J Rheumatol 1999; 26: 68-72.

6. Wong LP, Lee KC. A young lady with facial asymmetry: a case of lupus profundus. Hong Kong I Dermatol Venereol 2012; 20: 29-32.

7. Massone C, Kodama K, Salmhofer W, et al. Lupus erythematosus panniculitis (lupus profundus): clinical, histopathological, and molecular analysis of nine cases. J Cutan Pathol 2005; 32: 396-404.

8. Arai S, Katsuoka K. Clinical entity of lupus erythematosus panniculitis/lupus erythematosus profundus. Autoimmun Rev 2009; 8: 449-52.

9. Magro CM, Crowson AN, Kovatich AJ, et al. Lupus profundus, indeterminate lymphocytic lobular panniculitis and subcutaneous T-cell lymphoma: a spectrum of subcuticular T-cell lymphoid dyscrasia. J Cutan Pathol 2001; 28: 235-47.

10. Szczerkowska-Dobosz A, Olszewska B, Lemańska M, et al. Acquired facial lipoatrophy: pathogenesis and therapeutic options. Postep Derm Alergol 2015; 32: 127-33.

11. Goodfield M, Davison K, Bowden K. Intravenous immunoglobulin (IVIg) for therapy-resistant cutaneous lupus erythematosus. J Dermatol Treat 2004; 15: 46-50.

12. McArdle A, Baker JF. A case of "refractory" lupus erythematosus profundus responsie to rituximab. Clin Rheumatol 2009; 28: 745-6.

13. Tristano AG. Macrophage activation syndrome: a frequent but under-diagnosed complication associated with rheumatic diseases. Med Sci Monit 2008; 14: 27-36.

14. Granata G, Didona D, Stifano G, et al. Macrophage activation syndrome as onset of systemic lupus erythematosus: a case report and a review of the literature. Case Rep Med 2015; 2015: 294041; doi:10.1155/2015/294041.

15. Craig AJ, Cualing H, Thomas G, et al. Cytophagic histiocytic panniculitis - a syndrome associated with benign and malignant panniculitis: case comparison and review of the literature. J Am Acad Dermatol 1998; 39: 721-36.
16. Deji N, Sugimoto T, Fujimoto M, et al. Emergence of panniculitis and haemophagocytic syndrome in a patient with chronic systemic lupus erythematosus. Lupus 2007; 16: 363-5.

17. Vilaiyuk S, Sirachainan N, Wanitkun S, et al. Recurrent macrophage activation syndrome as the primary manifestation in systemic lupus erythematosus and the benefit of serial ferritin measurements: a case-based review. Clin Rheumatol 2013; 32: 899-904.

18. Takahashi K, Kumakura S, Ishikura H, et al. Reactive hemophagocytosis in systemic lupus erythematosus. Intern Med 1998; 37: 550-3. 